\title{
Essais
}

ESSAIS

Revue interdisciplinaire d'Humanités

$8 \mid 2016$

Erreur et création

\section{La lecture aberrante d'une inscription à Kasserine (Tunisie). Prospérité et chute d'une création par} erreur

Hernán González Bordas

\section{(2) OpenEdition \\ Journals \\ Édition électronique \\ URL : http://journals.openedition.org/essais/4943 \\ DOI : $10.4000 /$ essais.4943 \\ ISSN : 2276-0970 \\ Éditeur \\ École doctorale Montaigne Humanités}

Édition imprimée

Date de publication : 15 mars 2016

Pagination : 26-47

ISBN : 978-2-9544269-7-6

ISSN : $2417-4211$

Référence électronique

Hernán González Bordas, « La lecture aberrante d'une inscription à Kasserine (Tunisie). Prospérité et chute d'une création par erreur », Essais [En ligne], 8 | 2016, mis en ligne le 28 octobre 2020, consulté le 30 octobre 2020. URL : http://journals.openedition.org/essais/4943 ; DOI : https://doi.org/10.4000/ essais. 4943 


\title{
La lecture aberrante d'une inscription à Kasserine (Tunisie). Prospérité et chute d'une création par erreur
}

\author{
Hernán González Bordas
}

La tradition manuscrite est une source d'information importante concernant les textes épigraphiques et l'archéologie, autant pour l'Afrique Romaine que pour le reste de la Méditerranée antique en général. Aujourd'hui, en attendant d'hypothétiques nouvelles découvertes, les manuscrits déjà connus sont toujours utiles pour augmenter la liste des textes, les reconstituer et mieux connaître l'histoire de l'épigraphie. Cependant, un regard critique s'avère indispensable lors de la lecture de ces manuscrits. En effet, selon les auteurs, les contrefaçons peuvent être monnaie courante, mais aussi les erreurs. Si ces dernières se limitent normalement à de petites variantes de lecture des inscriptions, parfois elles peuvent être plus importantes, devenant difficiles à distinguer des falsifications, comme nous l'observerons dans les lignes qui suivent.

Tunis constituait au XVIII ${ }^{e}$ siècle une des villes les plus cosmopolites de la Méditerranée $^{1}$. Peuplée d'Arabes d'origine africaine et morisque ${ }^{2}$, de Turcs, de Berbères et de communautés juives d'origine africaine, elle accueillait aussi un milieu européen constitué de commerçants chrétiens, de juifs d'origine européenne, de consuls des principales nations, de religieux de différents ordres ainsi que de voyageurs de passage ${ }^{3}$. La présente étude se concentre sur quatre antiquaires issus de ces derniers groupes, intéressés, chacun à sa mesure, par les vestiges antiques : Francisco Ximenez, Gabriel de Mendoza, Joseph Carrillo et Jean André Peyssonnel. Ces protagonistes partagent des responsabilités dans la création et la transmission de la lecture d'une inscription provenant du mausolée des Flauii à Kasserine, à un tel point erronée qu'elle peut sembler une contrefaçon.

1 Voir, Paul Sebag, Tunis au XVII' siècle, Paris, L'Harmattan, 1989.

2 Rajah Yassine Bahri, "Les morisques en Tunisie un siècle après leur arrivée ", Cartas de la Goleta 2. Colloque international. Les morisques et la Tunisie. Expulsion, arrivée, impact et héritage. Beit el Hikma Carthage-Hannibal 13, 14, 15 novembre 2008. Tunis, p. 157-176.

3 Ahmed Saadaoui, "Les Européens à Tunis aux XVII et XVIII ${ }^{e}$ siècles ". Cahiers de la Méditerranée, 67, p. 61-84 (édition électronique), 2003. 
Notre but est de clarifier l'ensemble de ces responsabilités pour mieux comprendre les échanges d'informations entre les Européens de Tunis au XVIII ${ }^{e}$ siècle, mais aussi pour identifier la genèse de ce texte. En effet, les hypothèses avancées jusqu'à ce jour demeurent insatisfaisantes. Pour cela nous présenterons les protagonistes, puis nous analyserons les divers témoignages dans lesquels ce texte apparaît. Enfin une approche détaillée du journal inédit de F. Ximenez dévoilera des informations précieuses et inconnues sur les rapports entre ces antiquaires.

\section{Les antiquaires}

\section{Francisco Ximenez}

Né à Esquivias (Tolède) en 1685, F. Ximenez se décrit dans son journal ${ }^{4}$ comme myope ${ }^{5}$, caractéristique physique digne d'intérêt, comme nous le verrons, dans le cadre de notre recherche. Sa formation lui a conféré une bonne maitrise du latin ecclésiastique (on trouve dans son journal des lettres adressées au Saint-Siège en cette langue).

Faisant partie de l'ordre des Trinitaires ${ }^{6}$, il se rendit en Afrique du Nord pour s'occuper des captifs chrétiens et améliorer leur situation, intercédant dans leur rachat et s'occupant de leur retour à la chrétienté. Il resta une quinzaine d'années dans la Régence de Tunis (1720-1735), ce qui lui permit de rencontrer des voyageurs, savants et curieux et de faire treize excursions dans l'intérieur du pays ainsi que sur les côtes. Pendant ce temps, il écrivit son journal de façon quotidienne et avec de rares interruptions seulement, pour cause de maladies ou de petits accidents. Il copia dans ce journal un total de 244 inscriptions.

Les écrits de F. Ximenez n'ont pas encore été publiés. Ils ont seulement été utilisés par les rédacteurs du CIL pour le volume VIII. Les manuscrits (dixhuit volumes au total) contiennent le Diario de Argel et le Diario de Tunez, l'Historia del Reyno de Tunez ${ }^{8}$ et l'Historia de los Cartagineses. Ils sont conservés à la Biblioteca de la Real Academia de la Historia, à Madrid.

4 F. Ximenez, Diario de Argel et Diario de Tunez - voir infra-que nous citerons selon le volume, du vol. I au vol. VII.

5 F. Ximenez, vol. I, fol. 7 vo.

6 Et non pas de celui de la Merci, affirmation erronée qui se perpétue depuis la parution du Corpus Inscriptionum Latinarum (dorénavant CIL) VIII, avec la seule exception, à ce que nous savons, de Sophie Saint-Amans, Topographie religieuse de Thugga (Dougga), Pessac, Paris, Ausonius, de Boccard, 2004, p. 19, n 11.

7 Mis à part un texte qui porte essentiellement sur la fondation de l'hôpital de Saint Jean de Matha, publié par Ignacio Bauer (Colonia Trinitaria de Tunez, Tetuán,1934) et des notes qui sont attribuées à F. Ximenez par R. Thouvenot et publiées en 1938 : Raymond Thouvenot ("Notes d'un Espagnol sur un voyage qu'il fit en Tunisie », Revue tunisienne, n.s., IX, p. 313-322). Ouvrage qui, dans la présente étude, sera cité : vol. XII. 


\section{Gabriel de Mendoza et Joseph Carrillo}

Le journal de F. Ximenez constitue la source de la plupart des informations dont nous disposons concernant G. de Mendoza et J. Carrillo9. Malgré leur origine espagnole, ils sont assimilés à la communauté juive portugaise ${ }^{10}$. Au service du Bey, G. de Mendoza fit de J. Carrillo son collègue et son gendre. Le premier, originaire d'Alcalá de Henares ${ }^{11}$, n’a pas laissé d'ouvrages de sa plume, publiés ou inédits.

Quant aux origines du second, J. Carrillo, il serait né à Madrid selon son éditeur, John Locke ${ }^{12}$. Il arrive à Tunis en $1718^{13}$ pour se convertir au judaïsme ${ }^{14}$ et il se marie avec une des filles de G. de Mendoza. Dans les années 1730 , il conçoit un recueil d'inscriptions. Cette syllogé sera publiée par J. Locke en $1763^{15}$. Rédigée en latin, elle contient cent six textes accompagnés, pour la plupart, d'une présentation très succincte, voire inexistante. La grande majorité de ces textes provient d'informateurs. Par conséquent, J. Carrillo n'a pas observé la plupart des monuments épigraphiques qu'il décrit ${ }^{16}$.

F. Ximenez précise que J. Carrillo possédait une culture classique élevée. D’ailleurs, il transcrit un poème en latin composé par ce dernier pour l'hôpital en janvier $1730^{17}$.

Grâce au journal, on peut bannir, une fois pour toutes, l'orthographe "Carilos », trop souvent utilisée pour mentionner J. Carrillo ${ }^{18}$. La responsabi-

9 Pour un portrait général des deux personnalités, Clara Álvarez Dopico prépare un article qui sera publié sous peu : «Semblanza de los doctores Mendoza y Carrillo. A propósito de las afinidades intelectuales y la acogida de criptojudíos en el Túnez dieciochista ", Seminario de Estudios Árabo-Románicos. Colección Iberia \& Berbería, 2015.

10 Livournais d'origine portugaise, les Grâna passèrent dans la Régence de Tunis durant le XVII s siècle. Ils étaient appelés « juifs européens " par rapport à ceux qui, dans l'imaginaire des Tunisois, étaient établis depuis toujours (Ahmed Saadaoui, op. cit., paragraphes 4 et 5).

11 Vol. V, fol. $71 \mathrm{r}^{\mathrm{o}}$ (Diario de Tunez).

12 John Locke, « Roman Inscriptions of Tunis in Africa, copied about the Year 1730, by Dr Carilos, a native of Madrid, then physician to the Bey of Tunis, communicated by John Locke ", Philosophical Transactions 53, 1763, p. 211.

13 Vol. VII, fol. $182 \mathrm{r}^{\mathrm{o}}$ (Diario de Tunez).

14 Vol. V, fol. 8 vo (Diario de Tunez).

15 Voir note 12.

16 Les inscriptions sont organisées par sites. Dans les deux premières pages, des bordures de colonnes sont dessinées, de façon très schématique, au sein desquelles se trouvent quatre textes épigraphiques. Ces dessins, signés par J. Mynde Sc., sûrement un collaborateur de J. Locke, ne semblent pas provenir de la plume de J. Carrillo, ou alors il s'agit de réélaborations à partir des brouillons de ce dernier.

17 Vol. VII, fol. 239 vo. Voir infra.

18 Dans le CIL, VIII, p. XXIV-XXV, mais aussi dans des ouvrages récents comme JeanMarie Lassère et al., Les Flauii de Cillium, Rome, École Française de Rome, 1993 ou Jehan Desanges et al. (éd.), Carte des routes et des cités de l'est de l'Africa à la fin de l'antiquité, d'après le tracé de Pierre Salama, Turnhout (Belgique), Brepols, 2010, p. 25. 
lité de cette erreur revient à J. Locke qui publia les « Roman Inscriptions... ». En dehors du fait que le nom "Carilos » n'existe pas en espagnol, il faut tenir compte qu'en anglais on ne distingue pas un $r$ ni un $l$ simple d'un double. Étant donné que F. Ximenez l'appelle $D r$ Carrillo $^{19}$ en se référant bien à quelqu'un à qui il a fourni des inscriptions, et que ces inscriptions sont publiées dans le recueil mentionné, nous sommes certain qu'il s'agit du même individu. La transcription préférable est celle faite par F. Ximenez, donc Carrillo, un nom d'ailleurs très courant en espagnol. Nous verrons par la suite que ce n'est pas le seul problème d'identité qui lui a été associé : les rédacteurs du CIL, VIII ont pensé qu'il était la même personne que G. de Mendoza.

\section{Jean André Peyssonnel}

Médecin et naturaliste, J. A. Peyssonnel (1694-1759) appartenait à une famille de médecins de Marseille. Il poursuivit ses études au collège de l'Oratoire de cette ville, où, selon l'historienne Lucette Valensi, il s'est nourri d'antiquité romaine ${ }^{20}$. En revanche, le chercheur Charles Monchicourt estimait que " malheureusement, ses compétences en histoire, tant ancienne que moderne, sont très moyennes $»^{21}$. Lors d'un séjour à Paris pour solliciter la création d'une chaire de médecine navale à Marseille, requête qui lui fut refusée, le Roi lui accorda tout de même la possibilité de partir en Barbarie dans le cadre d'un voyage de recherches ${ }^{22}$. Des passeports et des lettres de recommandation pour les autorités locales furent alors mis à sa disposition.

J. A. Peyssonnel séjourna en Tunisie de mai 1724 jusqu'à la fin de l'année 1725. Son statut pendant ce voyage est difficile à définir. Il obtint des passeports pour aller mener des recherches en histoire naturelle. Officiellement, il était donc envoyé par le roi, à ses propres frais, pour recueillir des plantes. Or, pour contenter un de ses correspondants, l'Abbé Bignon membre de l'Académie des Sciences et de l'Académie des Inscriptions, il s'occupa aussi des antiquités qu'il trouvait au fur et à mesure de son itinéraire.

Nous possédons aujourd'hui deux ouvrages de J. A. Peyssonnel sur son voyage en Tunisie : les Lettres, rédigées pendant son séjour dans la Régence et éditées en 1838 par Adolphe Dureau de la Malle²3, ainsi que la Relation d'un

19 Entre autres dans vol. V, fol. $8 \mathrm{r}^{\mathrm{o}}$ et vo (cf. transcription infra).

20 Lucette Valensi (éd.), J. A. Peyssonnel. Voyage dans les régences de Tunis et d'Alger. Paris, La Découverte, 1987, p. 25.

21 Charles Monchicourt « Le voyageur Peyssonnel de Kairouan au Kef et à Dougga (août 1724) ", Revue Tunisienne, XXIII, 1916, p. 363.

22 Auguste Rampal, «Une relation inédite du voyage en Barbarie du médecin naturaliste marseillais Peyssonnel ", Bulletin de Géographie historique et descriptive, n 2, p. 324.

23 Adolphe Dureau de la Malle, Peyssonnel et Desfontaines. Voyages dans les régences de Tunis et d'Alger, Paris, 1838. 
voyage dans le royaume de Tunis et d'Alger par ordre du Roy en 1724, inédite ${ }^{24}$, trouvée à Avignon au début du XX $\mathrm{XX}^{\mathrm{e}}$ sielcle $^{25}$. Cette Relation, écrite très vraisemblablement entre 1725 et $1727^{26}$, présente à peu près le même contenu que les Lettres, mais elle suit une structure thématique plutôt que chronologique. Elle fournit de même quelques fragments nouveaux.

\section{La première erreur}

F. Ximenez écrit dans son Diario, en mars 1722, que G. de Mendoza et J. Carrillo lui rendent visite à Tunis et lui apportent la description des tours d'une ville du sud de la Régence dont ils ignorent le nom. Ces tours portent des inscriptions latines. F. Ximenez copie ces informations dans son Diario, en incluant la transcription d'une inscription avec des éléments suspects (fig. 1). Parmi ces éléments, on remarque le mélange éclectique entre les noms grecs Epamenondae, Agesilao et la mention de la "Reine de Sparte ", avec la formule typique des bulles papales ad perpetuam rei memoriam. Des réminiscences à l'histoire antique de Sparte sont ainsi combinées avec insouciance à des formules propres à la chancellerie pontificale. Cette transcription de G. de Mendoza et J. Carrillo constitue ce que nous avons appelé la « lecture aberrante ${ }^{27}$.

24 Sauf pour quelques fragments dans les articles cités d'A. Rampal et Ch. Monchicourt.

25 Auguste Rampal, op. cit., p. 317.

26 Ibid., p. 325.

27 Pour le choix de cette expression voir les conclusions en fin d'article. 


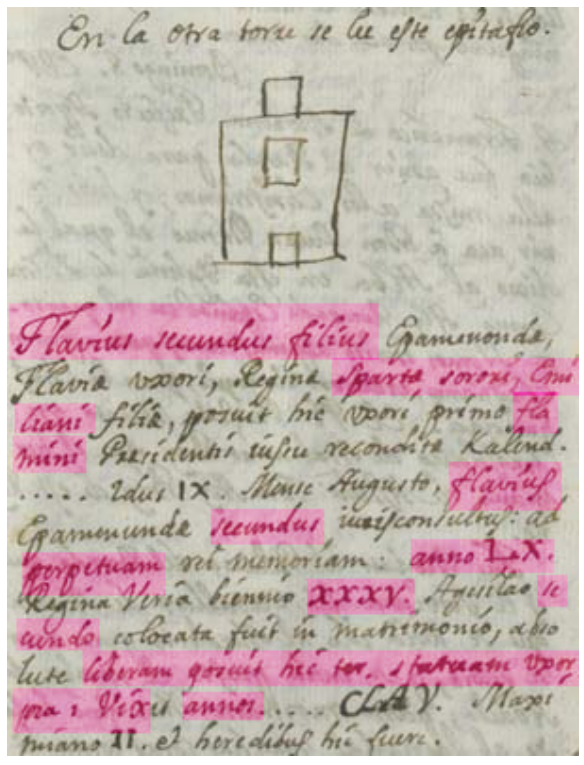

Fig. 1 : Première version du faux figurant dans le manuscrit de F. Ximenez. Les mots coïncidant avec CIL, VIII, 211 ont été soulignés par nous. Vol. V, fol. $8 \mathrm{r}^{\circ}-9 \mathrm{v}$, 6 mars 1722 .
C.I.L. VIII, 211.

1 T*FLAVIVS SE
CVNDVS FILIVS* FECIT

T*FLAVIO $*$ SECVN

5 DO *PATRI*PIO* $\mathrm{MIL} * \mathrm{AN} * \mathrm{XXXIII} *$ $\mathrm{VIX} * \mathrm{AN} * \mathrm{CX} * \mathrm{H} * \mathrm{~S} * \mathrm{E} *$ FLAVIAE VRBANAE* MATRI $*$ PIAE $*$ VIX

10 $\mathrm{AN} * \mathrm{CV} * \mathrm{H} * \mathrm{~S} * \mathrm{E}$ * $\mathrm{FL} * \mathrm{SECVNDAE} \mathrm{SO}$ RORI $\mathrm{P} * \mathrm{~V} * \mathrm{~A} * \mathrm{XX} * \mathrm{H} * \mathrm{~S} * \mathrm{E} *$ $\mathrm{T} * \mathrm{FL} * \mathrm{MARCELLO} * \mathrm{FRA} *$ $\mathrm{TRI} * \mathrm{P} * \mathrm{~V} * \mathrm{~A} * \mathrm{XX} * \mathrm{H} * \mathrm{~S} * \mathrm{E} *$ $\mathrm{T} * \mathrm{FL} * \mathrm{MARTIALI}$ FRATR $*$ $I * M I L * A * X I I * V * A * X X X V * H * S * E *$ $\mathrm{FL} *$ SPERATAE SORO* $\mathrm{RI} P * \mathrm{~V} * \mathrm{~A} * \mathrm{XXXVI} * \mathrm{H} * \mathrm{~S} * \mathrm{E} *$ AEMILIAE $*$ SEX $*$ FIL $*$ PACATAE*VXORI*PIAE FLAMINICAE*PERP* $\mathrm{VIX} * \mathrm{AN} * \mathrm{LIII} * \mathrm{H} * \mathrm{~S} * \mathrm{E} *$ $T * F L A V I V S * T *$ FILIVS *PAP*SECVNDVS *IPSE* FLAMEN PERP*VIX* $\mathrm{AN} * \mathrm{LX} * \mathrm{H} * \mathrm{~S} * \mathrm{E} *$

$\mathrm{FL} * \mathrm{~T} * \mathrm{FILIAE} * \mathrm{PACATAE} * \mathrm{FLA}$ MINICAE $* P E R P * C O L * T H E *$ LEPT*FIL *PIAE*FL *LIBERA MA* 30 TER STATVAM $*$ POSVIT * $V * A * X V * M * X * H * S * E *$ $\mathrm{F} * \mathrm{LIBER} A * \mathrm{~T} * \mathrm{FL} * \mathrm{SECVNDI}$ VXOR $* \mathrm{PIA} * \mathrm{VIX} * \mathrm{AN} * \mathrm{LXXXVIII}$ $* \mathrm{H} * \mathrm{~S} * \mathrm{E} *$

\section{(façade)}

Fig. 2 : L'épitaphe de T. Flauius Secundus et sa famille (CIL, VIII, 211), sur la façade S-O du Mausolée des Flauii. Les mots coïncidant avec le faux ont été soulignés en gris par nous. Dans Jean-Marie Lassère, op. cit., note 18.

En effet, il ne s'agit pas d'une invention ex nouo: G. de Mendoza et J. Carrillo étaient bel et bien devant une inscription latine réelle (fig. 2) lorsqu'ils ont fait cette lecture. Les fragments que nous avons soulignés en rose sont ceux qui ont permis de comprendre qu'il s'agit d'une transcription très particulière de CIL, VIII, 211. Cette inscription se trouve en effet dans la ville de Kasserine, sur le mausolée des Flanii (à la hauteur du A dans les fig. 3 et 4 ; 
$\mathrm{B}$ et $\mathrm{C}$ désignent d'autres épigraphes) ; le début du texte se situe à 8 mètres de hauteur et la fin à 5 mètres. Il s'agirait a priori d'une interpolation, c'est-à-dire, de l'ajout d'éléments inventés, lors de la transcription, insérés dans le texte provenant du monument. Ceci peut constituer un type de falsification, car même si seulement quelques éléments sont inventés le produit final reste un texte qui n'a jamais existé sur le monument épigraphique. Pourtant, s'il s'agit d'une falsification, les motivations sont difficiles à établir.

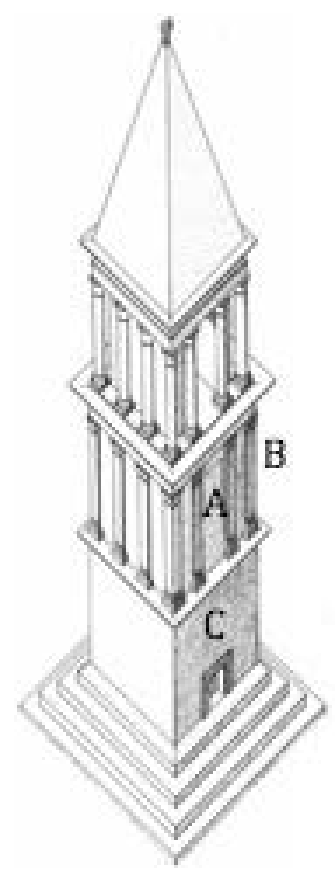

Fig. 3 : Restitution axonométrique du monument comme il devait être dans l'Antiquité, actuellement les colonnes du troisième niveau et le couronnement pyramidal sont manquants. Dans Jean-Marie Lassère, ibid.

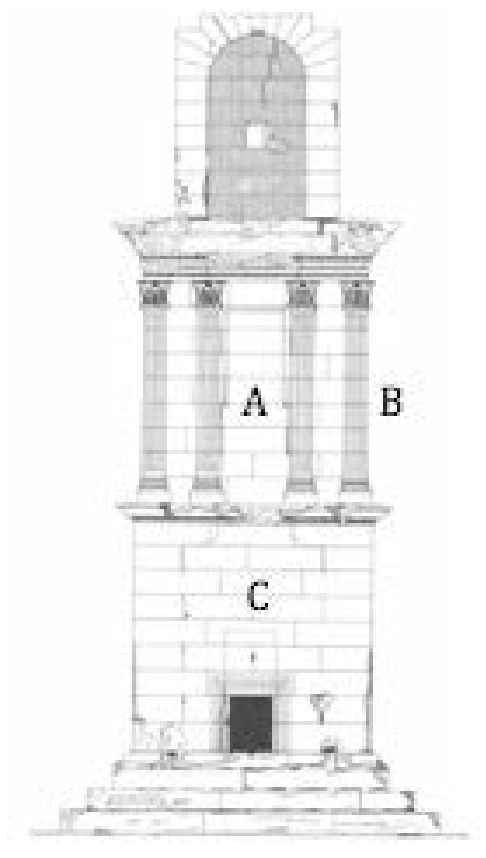

Fig. 4 : Dessin de la façade S-O du monument en son état actuel. Dans Jean-Marie Lassère, ibid.

\section{Une contrefaçon volontaire?}

Marc Mayer $^{28}$ considère qu'une inscription fausse peut être créée dans un manuscrit dans le but de donner une impression d'érudition sur un sujet historique quelconque : dans CIL, II, 412*, 413*, $414^{* 29}$, l'humaniste et faus-

28 Marc Mayer, L'art de la falsificació. Falsae inscriptiones a l'epigrafia romana de Catalunya. Barcelona, Institut d'Estudis Catalans, 1998.

29 CIL, II, 412* : Dianae inuictae | Augustae | conseruatr(ici) coloniae | Iuliae Fauentiae | Piae Augustae Barci $\mid$ nonensis $\mid P($ ublius) Pupius P(ublii) f(ilius) Trom(entina) Lati $\mid$ nus ueteranus leg III | Augustae Fauentiae | ex not(o) sol(uit). 
saire Pirro Ligorio cite ce qu'il considère comme le nom complet de la ville de Barcelone, commettant de la sorte à la fois une contrefaçon, aussi qualifiée de forgerie, et une méprise ${ }^{30}$.

Le but de cette forgerie peut également consister à prouver une hypothèse ou à légitimer la démonstration d'une théorie. C'est le cas des inscriptions interpolées par J. A. Peyssonnel pour indiquer la situation de la ville romaine de Sufetula (cf. infra). Cette motivation apparaît souvent directement liée à des localismes ou régionalismes : par exemple, lorsqu'un érudit souhaite glorifier sa petite patrie en apportant une inscription de son propre $\mathrm{cru}^{31}$. D'autres fois, il s'agit simplement d'étayer une légende (CIL, II, $421^{* 32}$ mentionne l'alliance d'un héros, Telongus Bachius, qui aurait combattu avec les Romains contre Hannibal lors de sa traversée des Pyrénées).

En dernier lieu, la falsification peut tout simplement être forgée pour embellir un recueil d'inscriptions avec un texte qui contient plus de substance que la norme, et qui peut donc attirer l'attention du lecteur (surtout du lecteur non-spécialiste), plus qu'une simple inscription funéraire. Bien entendu, dans la plupart des cas, le faux sera porteur d'informations remarquables pour son contenu textuel, mais souvent aussi pour ses caractéristiques formelles ${ }^{33}$.

Revenons maintenant à notre cas. Après une comparaison entre CIL, VIII, 211 et la lecture aberrante, ayant considéré les nombreuses similitudes, mais aussi les divagations fantaisistes empruntées au monde hellénique ou à la culture ecclésiastique, la volonté de tromper ne nous semble pas manifeste. En effet, tenant compte de la hauteur où est située l'épitaphe, il doit s'agir ici, selon nous, d'une négligence à laquelle s'ajoute un manque de respect pour l'authenticité du texte.

CIL, II, 413* : Genio Aug(usti) | conseruatori | coloniae Augustae | Iuliae Piae Fauen | tiae Barcinonesis | M(arcus) Gauius M(arci) f(ilius) Tromen(tina) | Salluus | (centurio) leg(ionis) III Aug(ustae) Fauent(iae) | Piae ex uot(o) sol(uit)

CIL, II, 414* : Virt(uti) cons(ulari) Augus(tae) | et coloniae Augustae | Iuliae Piae Fauentiae | Barcinonensis $\mid Q$ (uintus) Hirius Q(uinti) F(ilius) Trom(entina) Lepidus | speculator leg(ionis) III Augustae $\mid d($ edit) d(edicauit)

30 Il cite Colonia Iulia Fauentia Pia Augusta Barcino, le nom correct étant Colonia Iulia Fauentia Pia Barcino.

31 Georges Fabre et Marc Mayer, «Falsae et alienae : quelques aspects de l'application des critères d'E. Hübner à l'épigraphie romaine de Catalogne ", Épigraphie Hispanique. Problèmes de méthode et d'édition, Actes de la table ronde internationale du CNRS, Bordeaux, 1984, p. 181 et Mayer, op. cit., p. 13.

32 CIL, II, 421* : Telongo Bachio qui | Poeno exerc(itu) cum Hannib(ale) | in Ital(iam) transeun | te cum S(enatu) P(opulo)q(ue) R(omano) cum | factione reip(ublicae) amica | sensit Blandenses | statuam d(edit) d(edicauit).

33 Un texte qui sert à illustrer ce type est le sauf-conduit de César pour Cicéron : M(arcum) T(ullium) Ciceronem ob eius | eximiam uirtutem et | egregias animi sui do $\mid$ tes per uniuersum or $\mid$ bem terrarum saluum | esse iubeo $\mid$ C(aius) I(ulius) Caesar. Faux mentionné dans Joseph Finestres et de Monsalvo, Sylloge inscriptionum romanarum quae in Principatu Catalauniae vel exstant vel aliquando exstiterunt, Barcelone, 1762, p. 318, comme figurant en page 199 d'un recueil de Ponç d'Icard (XVI ${ }^{\mathrm{e}}$ siècle), probablement les Epigrammata Antiqua Vrbis Tarraconensis. 


\section{Diffusion et survivances de la lecture aberrante}

Comme en témoigne son Diario, F. Ximenez transmet deux ans plus tard, le 21 juin 1724, la transcription de J. Carrillo et de G. de Mendoza à J. A. Peyssonnel :

Ahier vino al hospital Monsr. Pessonel Medico [...] y le di unas inscriptiones que copia el Doctor Mendoza en una Ciudad arruinada de la Africa. ${ }^{34}$

En revanche, J. A. Peyssonnel, dans la troisième lettre, rédigée trois jours après le passage que l'on vient de citer de F. Ximenez, affirme :

Voici, à ce sujet, ce qui m’a été rapporté par le docteur Mendoze. ${ }^{35}$

J. A. Peyssonnel omet de façon délibérée l'intermédiation de F. Ximenez. Il s'agit d'un procédé habituel : présenter une inscription d'un informateur qui ne l'a pas observée ne fait que mettre en doute la crédibilité de l'inscription.

Un mois plus tard, J. A. Peyssonnel part pour une excursion dans le sud de la Régence. Il affirme dans sa Lettre Sixième ${ }^{36}$ être arrivé à Sbeïtla, près de Kasserine, et il insère dans son récit des inscriptions considérées comme provenant de cette ville (fig. 5).

Par la suite, F. Ximenez a accès au récit et aux inscriptions de J. A. Peyssonnel et, au mois de décembre, il part lui-même pour le sud et se rend à Sbeïtla. Une fois sur place, il y repère plusieurs inscriptions jusque-là inconnues, dont il réalise des copies fidèles ${ }^{37}$. En revanche, il ne trouve aucune des inscriptions reportées par J. A. Peyssonnel ; la description du site ne concorde pas non plus, ce qui lui fait croire un moment donné qu'il s'est lui-même trompé de lieu ${ }^{38}$.

34 «Hier est venu à l'hôpital M. Pessonel, médecin, [...] et je lui ai donné des inscriptions que copie le Docteur Mendoza dans une ville en ruines de l'Afrique. »

Vol. VI, fol. $61 \mathrm{r}^{\mathrm{o}}$.

35 Adolphe Dureau de la Malle, op. cit. p. 49. Après la description de la ville, il mentionne également trois textes, dont la fameuse transcription.

36 Adolphe Dureau de la Malle, op. cit., p. 119-120.

37 Vol. VI, fol. $145 \mathrm{r}^{\mathrm{o}}$ et suivants.

38 Vol. VI, fol. $144 \mathrm{v}^{\circ}$. 


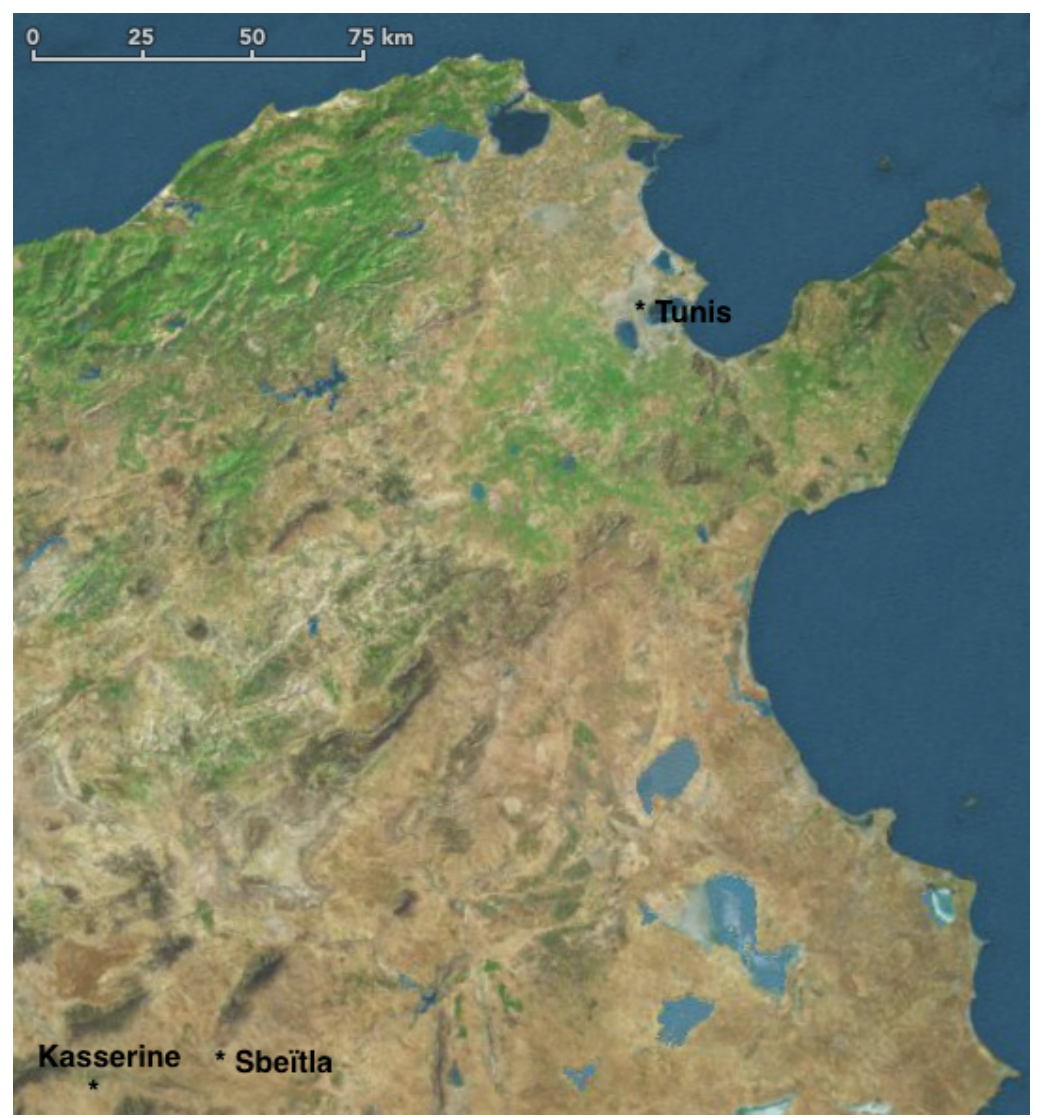

Fig. 5 : Situation de Kasserine et Sbeïtla par rapport à Tunis.

F. Ximenez se déplace ensuite à Kasserine, mais, pendant le trajet, il tombe de son âne et se déboîte le bras. Sur le site, il reconnaît les tours mentionnées par G. de Mendoza et J. Carrillo, bien qu'il se rende compte qu'il s'agit plutôt de mausolées. Au moment de décrire le mausolée des Flauii, il transcrit l'inscription CIL, VIII, 211. Mais en incluant aussi la transcription de J. Carrillo et de G. de Mendoza, il provoque une confusion autour de l'orientation des inscriptions. Il oriente le texte erroné vers le Midi et l'inscription réelle vers le Levant :

En la parte del norte encontramos un Mausoleo que todavia esta bien tratado es una torre alta como cinquenta palmos con algunas diminuciones en lo alto ay un nicho de aver avido alli alguna estatua. En la parte del mediodia esta escripto lo siguiente ${ }^{39}$ :

39 Dans le texte original la ponctuation est presque absente, ce que nous reproduisons dans notre traduction : "Du côté nord on trouve un Mausolée encore en bon état. Il s'agit d'une tour haute de quelque cinquante paumes avec quelques diminutions en haut on trouve une niche destinée à placer une statue. Du côté du midi il y a écrit ce qui suit ». 


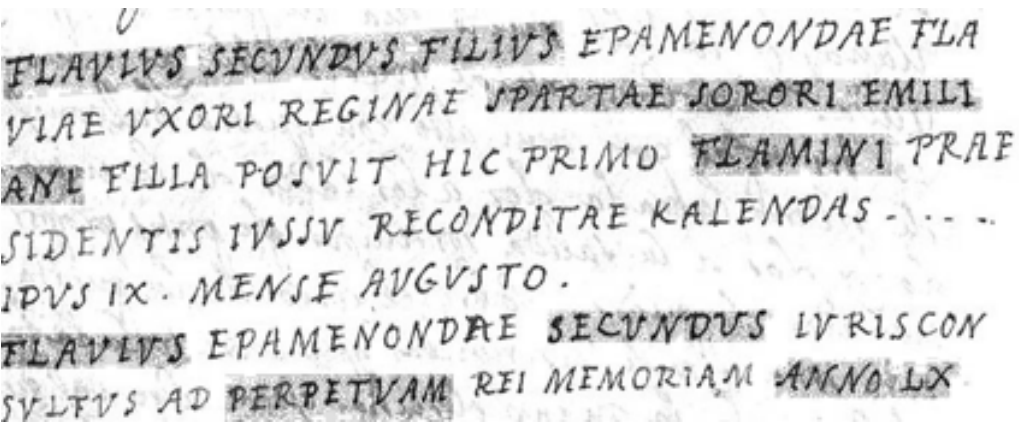

Fol. $147 v^{\circ}$.

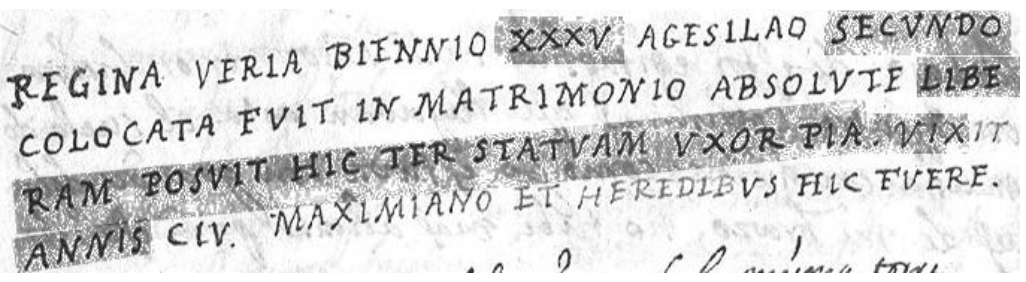

Fol. 148 ro.

Curieusement, cette version est bien différente de celle de 1722 (que F. Ximenez avait entre ses mains lorsqu'il décrivit le mausolée). Sur la plus ancienne, nous avons non seulement une mise en page distincte, mais aussi des terminaisons différentes pour certains mots comme Epaminonda ( $1^{\text {re }}$ ligne) Flauia, Regina, Sparta (2 $)$ Recondita (4') Epaminonda $\left(6^{\mathrm{e}}\right)$ annos $\left(11^{\mathrm{e}}\right)$. Plus frappant encore, cette version de 1724 a été expurgée de deux mots de l'ancienne : uxori ( $3^{\mathrm{e}}$ ligne) et $C L A V\left(11^{\mathrm{e}}\right)$, ce dernier devenant $C I V$, soit l'âge de l'uxor pia précédente. Cela fait un total de neuf différences.

Dans tous les cas, il s'agit toujours de la même lecture aberrante. L'inclusion du texte erroné constitue un point fondamental, car F. Ximenez n'est pas un interpolateur. Dans le cadre de nos recherches ${ }^{40}$, nous avons dépouillé tous ses ouvrages concernant les inscriptions de la Régence de Tunis et nous pouvons confirmer la bonne réputation dont il jouit depuis le CIL. F. Ximenez ne complète jamais les inscriptions avec des éléments inventés, même lorsqu'elles sont fragmentaires. Il mentionne ses sources lorsqu'il n'a pas observé lui-même les inscriptions. De plus, pour le cas qui nous occupe, ayant le monument devant lui, il n'a nul besoin de créer une forgerie.

40 Hernán González Bordas, Les inscriptions latines de la Régence de Tunis à travers le témoignage de F. Ximenez, sous la direction de Jérôme France. Thèse soutenue le 29 avril 2015 à l'Université Bordeaux Montaigne. 
Alors, pour quelle raison reprend-il la transcription de G. de Mendoza et de J. Carrillo ? Tout simplement par erreur, une erreur provoquée par sa bonne foi envers ses informateurs, cette même bonne foi qui lui avait fait croire qu'il n'était pas à Sbeïtla parce que ce qu'il voyait ne coïncidait pas avec la description de J. A. Peyssonnel. À cette erreur contribuèrent également sa myopie, qualité très ennuyeuse lorsqu'il s'agit de lire une inscription gravée à une hauteur considérable, et sa blessure au bras, dont les douleurs l'empêchèrent de rester le temps nécessaire sur le site et l'obligèrent à rebrousser chemin pour se faire soigner.

Cette erreur entraîne la survie de l'erreur initiale de G. de Mendoza et de J. Carrillo. Il s'agit d'un point fondamental, car la lecture aberrante, l'erreur, dépasse ainsi la première épreuve de véracité que constitue l'observation de F. Ximenez. Il fait preuve d'honnêteté d'esprit, néanmoins, il peine à contredire ses prédécesseurs.

Revenu en France, J. A. Peyssonnel rédige sa Relation entre 1726 et 1727 à partir du matériel compilé dans les Lettres. Dans cet ouvrage, il ajoute à la visite à Sbeïtla de sa Lettre Sixième une hasardeuse visite à Kasserine, où il inclut les inscriptions du journal de F. Ximenez avec, bien sûr, la transcription de G. de Mendoza et J. Carrillo. Cependant, cette version diffère beaucoup de celle qu'il avait présentée dans la troisième lettre, puisqu'il avait alors suivi la version transmise par F. Ximenez en 1722 tandis que cette fois-ci il suit la version de 1724. Quoi qu'il en soit, l'erreur continue d'être diffusée, l'inscription circule en France grâce aux Lettres et à la Relation de J. A. Peyssonnel.

F. Ximenez contribue également à la propagation de l'erreur. Si le Diario reste un texte à usage personnel, il inclut la lecture aberrante dans un recueil que nous avons nommé syllogé de Nîmes ${ }^{41}$. Il s'agit d'un recueil d'inscriptions, fruit de la compilation du matériel épigraphique lors de ses excursions dans la Régence jusqu'en 1726. Ce recueil est adressé à Scipione Maffei ${ }^{42}$, un des épigraphistes les plus marquants du moment, auteur d'un important corpus ${ }^{43}$ et d'un traité d'épigraphie ${ }^{44}$ où il prône la nécessité de l'autopsie - c'est-à-dire l'observation directe - des inscriptions.

41 Car elle est conservée aujourd'hui à la Bibliothèque Municipale de Nîmes, manuscrit MS0103_2-6 (fol. $124 \mathrm{r}^{\circ}$ ). Voir notre thèse citée dans la note précédente pour plus de détails.

42 Vérone, 1675-1755. S. Maffei avait conçu le projet épigraphique - échoué - de réunir toutes les inscriptions latines de l'Empire romain (Alfredo Buonopane, "Il Prospectus Vniversalis collectionis di Scipione Maffei e la nascita della scienza epigrafica ", Scipione Maffei nell'Europa del Settecento. Atti del Convegno - Verona 23-25 settembre 1996, Verona, p. 659.) plus d'un siècle avant le $C I L$.

43 Scipione Maffei, Museum Veronense, hoc est, Antiquarum inscriptionum atque anaglyphorum collectio : cui Taurinensis adiungitur et Vindobonensis : accedunt monumenta id genus plurima nondum vulgata, et ubicumque collecta, 1749, Vérone.

44 Scipione Maffei, Ars Critica Lapidaria, Lucca, 1765. 
De ce recueil, Sc. Maffei tire plusieurs inscriptions pour son Museum, mais la plupart sont écartées, lecture aberrante incluse. Sur le recueil ximénézien, il note pour celle-ci : «inedita ». Étant donné que son ouvrage n'était pas un corpus exhaustif d'inscriptions, nous ne pouvons pas savoir si Sc. Maffei la retenait pour exacte ou la considérait comme fausse. Il est très probable qu'il eut du mal à accepter que F. Ximenez se soit trompé, tenant compte de sa bonne opinion à son égard ${ }^{45}$. La validation de F. Ximenez confere ainsi une aura de vérité à l'erreur.

Curieusement, F. Ximenez lui-même aurait pu empêcher cette propagation. Après avoir rédigé la syllogé, à la fin des années 1720, il travaille sur son Historia del Reyno de Tunez. Il s'agit d'un ouvrage écrit dans son bureau, à Tunis, où il organise tout le matériel recueilli dans son Diario et le présente par chapitres organisés selon des subdivisions géographiques. Sans les incommodités des excursions - cas du Diario - ou les urgences pour terminer un recueil qui doit être envoyé - cas de la syllogé de Nîmes -, F. Ximenez élimine la lecture aberrante. Se rend-il compte qu'il s'agit de la même inscription que CIL, VIII, 211 ou bien juge-t-il qu'il n'y a pas assez d'espace sur la façade du mausolée pour inclure une telle inscription? Quoi que l'on réponde, le texte était déjà diffusé.

En dernier lieu, J. Carrillo lui-même, on l'a vu, rédige une syllogé qui est publiée trente ans plus tard en Angleterre. Bien entendu, la lecture aberrante est incluse puisque J. Locke, l'éditeur, ne porte aucune critique sur le recueil et le reproduit tel quel. L'erreur se propage ainsi dans trois pays européens.

\section{L'évolution dans la science épigraphique et la détection de l'erreur}

À la fin du XIX ${ }^{\mathrm{e}}$ siècle, les responsables du CIL qui dépouillent les manuscrits pour la rédaction du Corpus, éliminent la transcription de G. de Mendoza et J. Carrillo. Que s'est-il passé entre ces deux périodes pour que l'erreur soit expurgée?

Rappelons qu'à la fin du XVIII siècle la discipline épigraphique était entrée dans les parcours d'études comme une spécialité auxiliaire de la chronologie et de la géographie autant dans la péninsule italique que dans le Royaume de Prusse ${ }^{46}$. Francesco Antonio Zaccharia et surtout Bartolomeo Borghesi font fonction de charnière entre le siècle de Sc. Maffei et celui de Theodor Mommsen (fondateur du CIL), dont $\mathrm{B}$. Borghesi fut le précepteur.

À partir du moment où l'étude des inscriptions était, avant tout, perçue comme un moyen de datation, une inscription contenant des références à Epaminondas ou à la reine de Sparte, mêlées à des formules comme ad perpe-

45 Il l'appelle « eximius auctor» dans son Museum CCCCLV.

46 Ida Calabi Limentani, Scienza epigrafica. Contributi alla storia degli studi di epigrafia latina, (Epigrafia e antichità, 28), Faenza, Fratelli Lega, 2011, p. 345-347. 
tuam rei memoriam, ne pouvait plus être tolérée. Deuxièmement, le projet du CIL exigeait que les meilleurs épigraphistes, ceux formés avec ou par Th. Mommsen, se rendissent sur les sites pour observer les inscriptions. C'est ainsi que G. Wilmanns constata enfin à Kasserine, devant le mausolée des Flauii, que l'inscription en question n'avait jamais existé.

Néanmoins, ces épigraphistes à part la lecture aberrante, dans les Additamenta ${ }^{47}$, mais, n’ayant pas lu le Diario de F. Ximenez en détail, le stemma qu'ils y proposent pour la transmission est incorrect.

\section{Les hypothèses sur la transmission}

Quelles ont été les opinions des spécialistes, depuis Gustav Wilmanns, auteur principale du CIL, VIII, et Th. Mommsen jusqu'à nos jours, sur les voyageurs que l'on vient de présenter et sur leur rapport avec le mausolée des Flauii et la lecture aberrante?

Dans les Additamenta, les rédacteurs du CIL décrivent la lecture aberrante comme suit : "Inscriptionis 11 exemplum interpolatum uel, si magis placet, ficticia inscriptio $»^{48}$. Dans le commentaire, ils signalent qu'un individu appelé Mendoza ou docteur Mendoza a communiqué ce texte à J. A. Peyssonnel, qui l'aurait lui-même présenté pour la première fois dans la troisième de ses Lettres, adressée à l'Abbé Bignon ${ }^{49}$ et datée du 24 juin 1724.

Ensuite, ayant eu accès au recueil déjà mentionné «Roman Inscriptions... " ", et y ayant trouvé des copies identiques à celles que J. A. Peyssonnel disait être communiquées par G. de Mendoza, les rédacteurs du CIL ont pensé que ce dernier et J. Carrillo étaient la même personne ${ }^{51}$. Enfin, les auteurs du CIL indiquent aussi que F. Ximenez ajouta la transcription au fol. $147 \mathrm{du}$ premier volume du Diario, soit en date du 15 décembre 1724.

47 CIL, VIII, 2, p. 925.

48 "Reproduction altérée de l'inscription 211 ou, si l'on préfere, inscription fictive ».

49 Adolphe Dureau de la Malle, op. cit., p. 50-51.

50 John Locke, op. cit.

51 On a vu supra que cela n'a pas de sens, le Diario de F. Ximenez prouve qu'ils sont parents. 


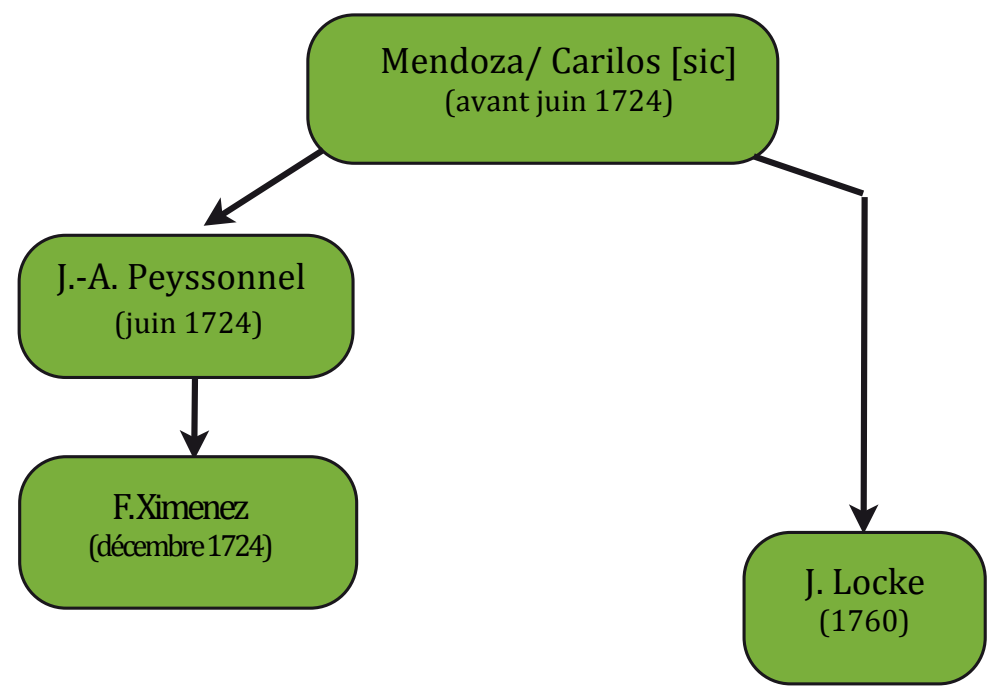

Tradition manuscrite de l'exemplum interpolatum de CIL, VIII, 211 selon le CIL.

Les rédacteurs du $C I L$, VIII ont succinctement commenté les liens existants entre les voyageurs de cette époque en portant le soupçon sur G. de Mendoza, comme nous venons de l'analyser, et sur J. A. Peyssonnel comme nous le verrons ensuite.

Dans la page XXV de l'Auctorum recensus de ce même volume du CIL, les auteurs présentent J. A. Peyssonnel en ces termes : "leuitatis autem summae adeoque fraudi proximae fuisse $~^{52}$. Ils évoquent par ailleurs F. Ximenez en page XXIV, mais avec davantage de considération : « ita reliquis longe praestat et copia et praestantia exemplorum, crediderimque uere Ximenezium primum fuisse, qui prouinciae Proconsulari [...] in re epigraphica aliquem locum dederit " ${ }^{53}$.

Un siècle plus tard, J. M. Lassère propose une autre possibilité pour la création de ce qu'il a appelé l'" inscription fantaisiste ". Étant donné que F. Ximenez était escorté par des chrétiens réduits en esclavage lors de ses voyages, l'historien avance l'hypothèse que ce sont ces derniers qui ont créé la forgerie : " Je suis porté à supposer que l'inscription fantaisiste [...] est [...] une copie de CIL, VIII, 211 faite, parallèlement à celle du religieux par un de ses compagnons chrétiens, instruit, mais peu entraîné à la lecture des pierres gravées, et de surcroît exagérément facétieux. Ximenez aurait conservé la fiche

52 «... il fut d'une très grande légèreté et même proche de la fraude ».

53 "Il est donc de loin au-dessus des autres (c'est-à-dire des autres voyageurs du XVIII ${ }^{e}$ siècle) autant pour la quantité que pour la qualité de ses copies, et je serais porté à croire effectivement que F. Ximenez fut le premier qui donna à la Proconsulaire une place dans les études épigraphiques ". 
dans son dossier... ${ }^{54}$. En parallèle à l'inscription fantaisiste, il y aurait donc la transcription fidèle établie par F. Ximenez lui-même.

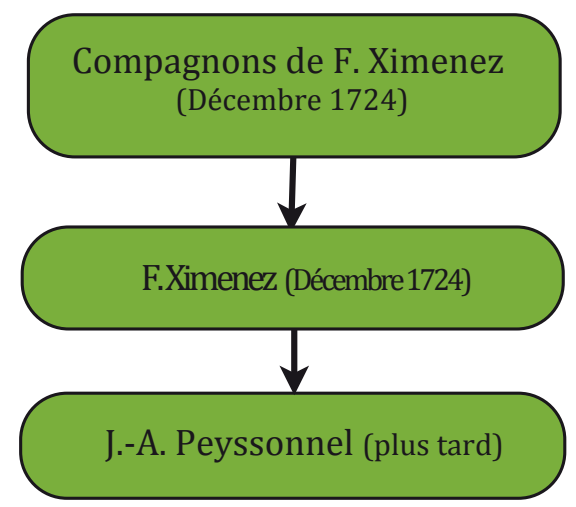

Tradition manuscrite de l'« inscription fantaisiste " selon J. M. Lassère.

Bien que très intéressante, cette hypothèse est contredite par plusieurs informations que nous avons précédemment mentionnées, notamment celles relatives au récit du Diario de mars 1722, à la chronologie du récit de la Lettre Troisième de J. A. Peyssonnel ou encore aux excursions de F. Ximenez.

\section{L'invention de J. A. Peyssonnel d'une excursion à Sbeïtla et Kasserine}

En analysant un épisode du parcours de J. A. Peyssonnel entre Kairouan et Le Kef, les historiens Ch. Monchicourt puis N. Duval ont confirmé les critiques faites à son sujet dans le CIL. En effet, ils ont établi qu'il ne s'était jamais rendu à Sbeïtla, pas plus qu'à Kasserine, et que les rapports qu'il fit sur ces sites ne seraient rien d'autre que le fruit de son imagination fertile et, dans une moindre mesure, du travail d'informateurs auxquels il fit appel.

Plus précisément, en s'appuyant sur le texte des Lettres ${ }^{55}$, Ch. Monchicourt ${ }^{56}$ a démontré que J. A. Peyssonnel avait inventé son voyage à Sbeïtla, ville très éloignée de son itinéraire, et avait étoffé la description de la ville avec des copies d'inscriptions interpolées qui n'ont jamais été retrouvées ${ }^{57}$.

54 Jean Marie Lassère et alii, op. cit., p. 15.

55 Lettre Sixième à l'Abbé Bignon datée du 16 août 1724, Adolphe Dureau de la Malle, op. cit., p. $119-120$.

56 Charles Monchicourt, op. cit., p. 358-361.

57 Dont CIL, VIII, 233 *IMP. CAES. AVG. | [---]|[---]|SVFFETVLENTIVM HANC|[---] AEDIFICAVERVNT |ET DD PP qui prétend donner crédibilité à son passage par Sbeïtla. Néanmoins l'erreur orthographique le dénonce, puisque la ville ancienne est Sufetula avec un seul $<\mathrm{f}>$. 
Après son retour en France, lors de la rédaction de la Relation (entre 1725 et 1727), J. A. Peyssonnel ajouta à la prétendue excursion à Sbeïtla de ses Lettres (août 1724) une autre visite inventée de toutes pièces, dans la ville de Kasserine cette fois. Dans cette Relation, il adjoignit des inscriptions et modifia également quelques descriptions du site de Sbeïtla par rapport au premier récit ${ }^{58}$.

Comme l'a démontré N. Duval ${ }^{59}$, J. A. Peyssonnel s'était inspiré pour sa Relation des descriptions du Diario que F. Ximenez ${ }^{60}$ avait élaborées lors de son passage dans ces contrées en décembre 1724.

Mais il est important de rappeler, comme nous l'avons vu, que bien qu' ignorant sa localisation géographique, J. A. Peyssonnel connaissait le texte erroné à Tunis déjà, lors de la rédaction de la troisième lettre (juin 1724), deux mois avant sa prétendue excursion. Selon lui et selon le $C I L$, le texte lui fut transmis par G. de Mendoza (or nous avons vu que cela n'est pas tout à fait vrai).

Dans la Lettre Sixième (août 1724), relatant le voyage entre Kairouan et Le Kef et inventant la visite à Sbeïtla, J. A. Peyssonnel ne mentionna pas ledit texte. Plus $\operatorname{tard}^{61}$ il le rajouta dans sa Relation (fin 1725 - début 1727), dans la partie du texte consacrée à la visite inventée à Kasserine. À ce moment ${ }^{62}$, il avait pu lire le rapport sur Sbeïtla et Kasserine du Diario de F. Ximenez (décembre 1724) et inclure dans son nouveau récit les informations réelles figurant dans celui de F. Ximenez ${ }^{63}$. Comme le lecteur l'aura bien observé, J. A. Peyssonnel se sert d'une erreur d'autrui pour donner crédibilité à son passage inventé sur Kasserine.

\section{G. de Mendoza ou J. Carrillo?}

Une dernière question reste à résoudre : fut-ce l'informateur de F. Ximenez, c'est-à-dire G. de Mendoza lui-même, qui altéra CIL, VIII, 211 au point de la transformer comme on l'a vu?

Divers indices nous portent à croire que ce fut plutôt son gendre, Joseph Carrillo. En premier lieu, celui-ci est proche du transmetteur de l'inscription, son beau-père G. de Mendoza. Deuxièmement, il est l'auteur du

58 Voir le parcours des excursions inventées selon Noël Duval, «La solution d'une énigme : les voyageurs Peyssonnel et Giménez à Sbeitla en 1724 ", Bulletin de la Société nationale des antiquaires de France (2 juin 1965), 1965, p. 113-132.

59 N. Duval, op. cit.

60 Ch. Monchicourt, de son côté, soupçonnait que ces inventions provenaient d'« un petit noyau de personnes enclines à procurer à un nouveau débarqué pas mal d'éclaircissements vrais ou inventés sur les choses de la Régence » et, entre eux, il distingue le docteur Mendoza. Charles Monchicourt, op. cit., p. 360.

61 Cf. infra.

62 Ils sont en correspondance en mars 1725, par exemple (vol. VI, fol $168 \mathrm{v}^{\circ}$ ).

63 Voir les deux tableaux en fin d'article. 
recueil épigraphique publié par J. Locke ${ }^{64}$. Le dernier indice est encore apporté par le Diario de F. Ximenez. Il y est mentionné65 qu'en date du 5 décembre $1730 \mathrm{~J}$. Carrillo rédige le poème suivant en hommage à l'hôpital fondé par les trinitaires dont F. Ximenez est l'administrateur :

De Nosocomio PP. Trinitarionum I Tunetino ${ }^{66}$

Sic Hispane salum Fratrum succensus amore undiuagum sulcas, nec tepes ignis aqua?

Non satis est, miseros, Nomadum relevasse catenis sed pietas agris prop<e $>$ ret arsa domus

5 Barbaries stupuit, posuereque ferocia Poeni corda volente Deo dum domus alma calet. Iupiter ulterius si uult descendere Olimpo, et cupiat, Poenos adire cassas; Iam reperire queat quantisper numine dignum

10 hospicium sanxit, quod peregrinus amor. Nec mirere : salus proles Iovis hospitet alma cum semper, quid ni sit Pater hospes ibi?

Il s'agit de six distiques élégiaques contenant peu d'erreurs en ce qui concerne la métrique. De plus, on doit ajouter une réminiscence virgilienne (v. 5-6) et deux autres (v. 1 et 9) provenant de recueils d'écrivains humanistes espagnols ${ }^{67}$. Le fragment virgilien...]que ferocia Poenil corda uolente Deo[... (Énéide, I, 302-303) ne signifie pas nécessairement que J. Carrillo ait lu le poète de Mantoue. En d'autres termes, il ne possédait pas nécessairement une culture classique par voie directe, les réminiscences virgiliennes ayant été les plus copiées et réutilisées depuis l'antiquité.

Dans tous les cas, nous avons affaire à quelqu'un qui a non seulement lu la poésie en langue latine, ce qui n'est pas étonnant pour un lettré de cette époque, mais qui est aussi capable de composer un texte métrique en latin. De même, le goût du poème qui mélange des références à la mythologie grécoromaine (v. 7 et 11) avec des ethnonymes et des toponymes (v. 1, 3, 5, 8) nous rappelle la mention des rois thébains et spartiates présents dans la lecture aberrante de CIL, VIII, 211.

64 J. Locke, op. cit.

65 Vol. VII, fol. $239 \mathrm{v}$.

66 Nous nous sommes permis de corriger ce texte qui comportait, en plus des difficultés de lecture, quelques erreurs d'orthographe dues soit à J. Carrillo soit à F. Ximenez.

67 Pour v. 1 SS. PP Toletanorum quotquot extant opera S. Eugeni. Pour v. 9 voir Vicente Ximeno, Escritores del Reyno de Valencia, chronologicamente ordenados desde el año M.CC.XXXVIII. de la Christiana Conquista de la misma Ciudad, hasta el de M.DCC.XLVII, Valencia, 1747 : F. Tarrega, V. Mariner. 


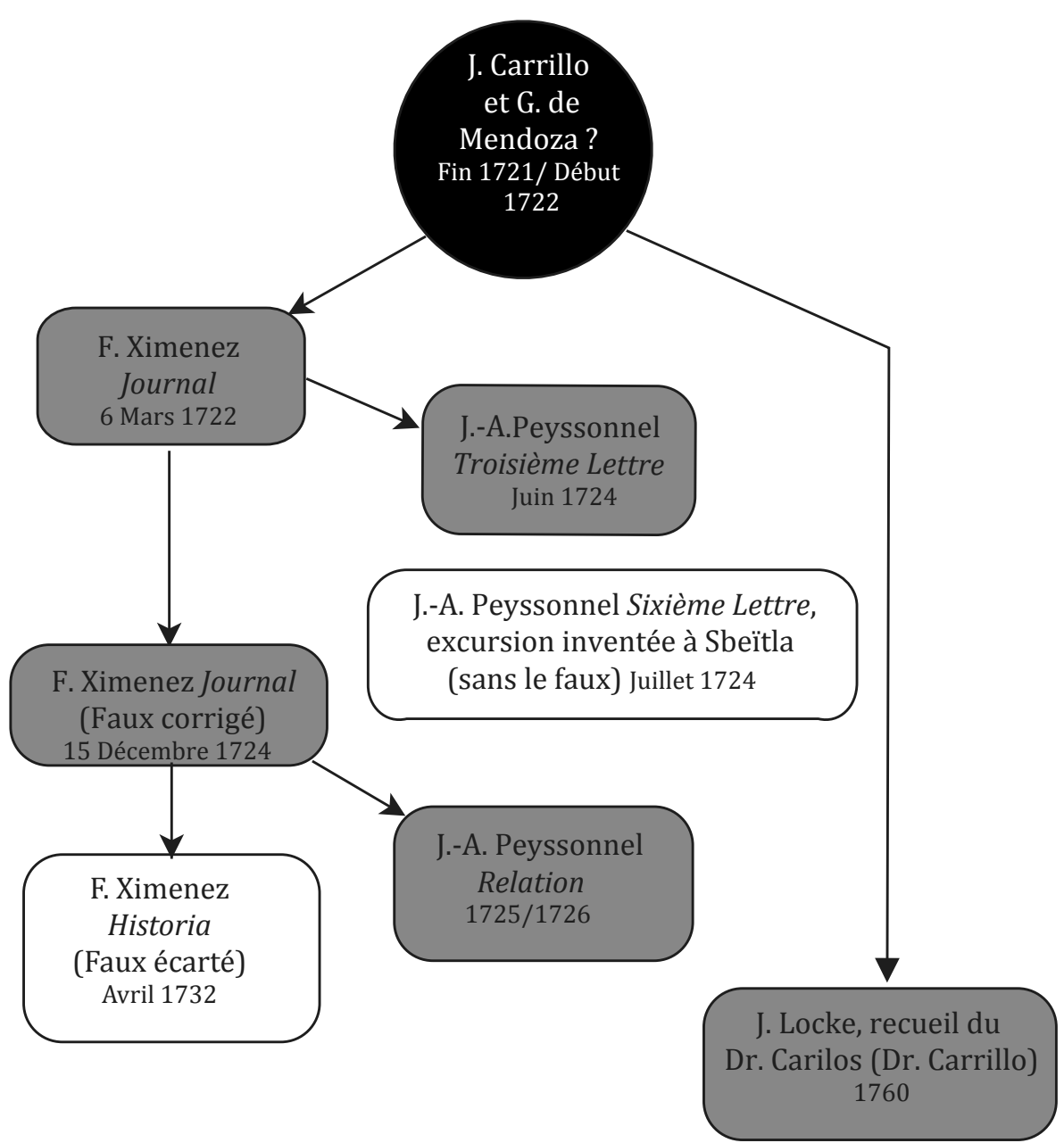

Tradition manuscrite de la lecture aberrante de CIL, VIII, 211 selon nous. 


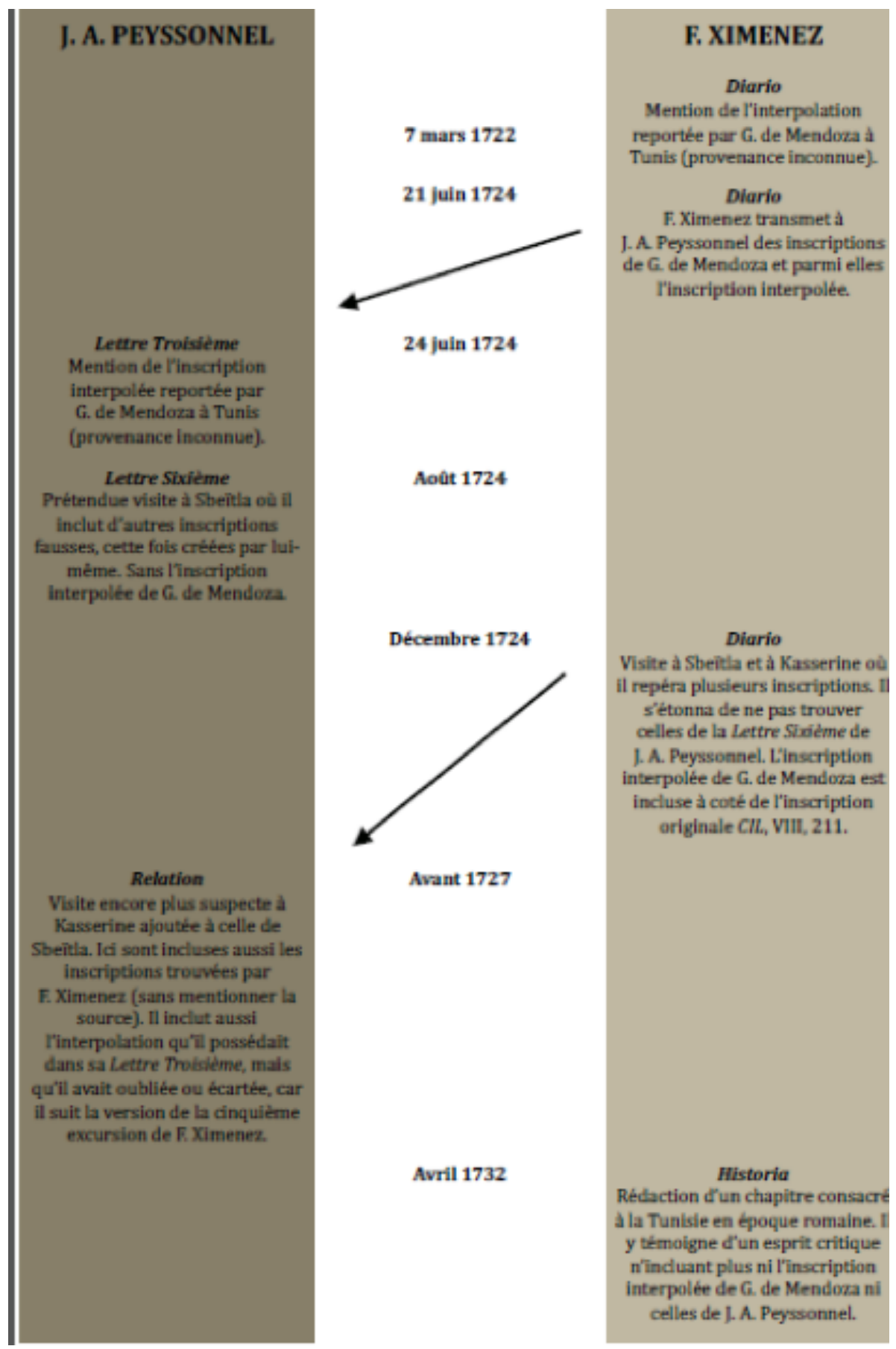

Chronologie des emprunts entre J. A. Peyssonnel et F. Ximenez. 


\section{Conclusions}

Afin d'illustrer les difficultés auxquelles l'historien doit faire face lors de l'usage des manuscrits, nous avons choisi un cas exceptionnel dans l'histoire de l'épigraphie. Erreur fantaisiste ou lecture aberrante, l'idée - et l'étymologie - derrière ces dénominations reste la même : il s'agit d'une erreur. Ensuite, la lecture aberrante de l'épitaphe du monument des Flanii acquiert une crédibilité grâce à une succession d'erreurs postérieures liées à la transmission du texte. C'est un enchaînement de ce type qu'illustre notre exemple et non une vraie volonté de falsifier. Les contrefaçons, comme nous l'avons vu, se justifient par des motifs précis qui sont extérieurs à l'objet de notre étude. En revanche, dans ce cas-ci l'impossibilité de voir le texte complet de l'inscription amène à simplement noter les trois ou quatre mots identifiables et quelques caractères isolés. Le reste est constitué de formules latines, noms propres ou mots qui évoquent l'antiquité.

Cet exemple montre, en premier lieu, les extravagances auxquelles conduit la lecture d'une inscription par une personne dont les notions en épigraphie latine sont approximatives (J. Carrillo) dans des conditions d'observations difficiles. Deuxièmement, bien qu'ils soient honnêtes (F. Ximenez), les antiquaires ont manqué d'esprit critique. À cela s'ajoutent les difficultés évoquées ce qui a parfois pu les amener à donner foi à des témoignages fantaisistes.

Ce manque d'esprit critique ajouté à l'absence de scrupules, dont certains peuvent faire preuve lorsqu'ils utilisent les témoignages d'autrui, conduit à des situations où l'erreur est réutilisée (J. A. Peyssonnel). Paradoxalement, cet abus servira à confirmer les méfiances des chercheurs à l'égard de J. A. Peyssonnel.

F. Ximenez écarte finalement cette lecture de son Historia. Il corrige ses relevés d'inscriptions chez lui, alors qu'il pourrait le faire sur place. Nous en déduisons qu'il fait plus confiance à son analyse attentive a posteriori qu'à l'examen visuel du monument. Se concentrant sur le texte en lui-même plutôt que sur l'inscription dans son ensemble, F. Ximenez procède en tant qu'analyste et correcteur de textes, au moins en ce qui concerne l'inscription qui nous occupe. Il faut donc dorénavant tenir compte de ce constat lorsqu'on se servira de son Historia pour la recherche en épigraphie latine.

Cette lecture aberrante a voyagé à travers les pays et les époques. Elle a été publiée en Angleterre, lue par des savants à Paris, puis analysée avec réticence à Vérone. Finalement, elle a été détectée, mais les épigraphistes contemporains ont peiné à retracer son parcours parce que toutes les sources n'avaient pas été exploitées.

Hernán González Bordas

UMR 5607 AUSONIUS

Université Bordeaux Montaigne

Hernan.Gonzalez-Bordas@u-bordeaux-montaigne.fr 


\section{Résumé}

Cet article plonge dans le milieu des antiquaires amateurs d'épigraphie du début du XVIII' siècle. dans la Régence de Tunis. Il explore la façon dont est créé, par des antiquaires aux connaissances limitées et dans des circonstances difficiles, un texte fantaisiste à partir de la mauvaise lecture d'une inscription. Ensuite, la tradition manuscrite de cette lecture a été retracée afin de mettre en évidence le processus de légitimation de ce témoignage erroné, qui fut écarté définitivement lors de l'avènement du CIL, œuvre fondatrice de l'épigraphie contemporaine.

\section{Mots-clés}

Antiquaires, XVIII siècle, Épigraphie latine, Tradition manuscrite, Régence de Tunis.

\section{Abstract}

This paper dives into the world of amateur epigraphists and antiquarians of the first part of the 18th century during the Tunis Regency. It shows how a fanciful text is created by antiquarians with limited knowledge and dealing with difficult conditions, from the misunderstood reading of a Latin inscription. Afterwards, we have tracked down the manuscript tradition of this reading in order to show the legitimization process of this misled evidence, which is definitely removed with the arrival of CIL, a founding work of the contemporary epigraphy.

\section{Keywords}

Antiquarians, 18th century, Latin epigraphy, Manuscript tradition, Regency of Tunis. 